\title{
Salvage surgery for head and neck cancer: a plea for better definitions
}

\author{
Alvaro Sanabria $\cdot$ Luiz P. Kowalski • Ashok R. Shaha • Carl E. Silver • \\ Jochen A. Werner $\cdot$ Magis Mandapathil $\cdot$ Robert P. Takes $\cdot$ Primož Strojan • \\ Alessandra Rinaldo $\cdot$ Alfio Ferlito
}

Received: 23 January 2014 / Accepted: 27 January 2014 / Published online: 15 February 2014

(C) Springer-Verlag Berlin Heidelberg 2014

\section{Introduction}

The term "salvage (or 'rescue') surgery" has been used to refer to surgical treatment after failure of initial treatment in various scenarios including treatment of delayed neck metastasis, recurrent primary tumors, or even lung metastasis. The first reference to "salvage surgery" for oncologic treatments found on PubMed was to surgical treatment for lack of response after adequate management of primary tumor with radiotherapy, published in 1965 [1]. In the treatment of head and neck cancer, the first reports from the 1970s refer to "salvage" in the form of a laryngectomy or pharyngolaryngectomy following radiotherapy [2, 3]. This concept was defined from trials which offered exclusive radiotherapy or radiochemotherapy $[4,5]$ for patients

This paper was written by members and invitees of the International Head and Neck Scientific Group (www.IHNSG.com).

\section{A. Sanabria}

Oncology Unit, Department of Surgery, Universidad de

Antioquia, Universidad de La Sabana, Hospital Pablo Tobón

Uribe, Medellín, Colombia

\section{P. Kowalski}

Department Otorhinolaryngology-Head and Neck Surgery, Centro de Tratamento e Pesquisa Hospital do Cancer A.C. Camargo, São Paulo, Brazil
A. R. Shaha
Head and Neck Service, Memorial Sloan-Kettering Cancer
Center, New York, NY, USA
C. E. Silver
Departments of Surgery and Otolaryngology-Head and Neck Surgery, Montefiore Medical Center, Albert Einstein College of Medicine, Bronx, NY, USA

with advanced head and neck tumors. Such patients will have two different outcomes: many will achieve a complete response after the non-surgical therapy and will not need further treatment, while some of them will have a partial response which will need a complement of the original treatment, to "rescue" them from the failure of the first treatment. However, with the introduction of newer treatment protocols, such as organ preservation schemes, and with knowledge of the outcomes of most tumors initially submitted to non-surgical therapies, the indications for "salvage surgery" have increased, but without a concomitant evolution of the term. Since the first description of salvage surgery after radio(chemo)therapy protocols, the number of publications related to the subject have increased dramatically. Salvage surgery is no longer limited to patients who failed radiotherapy or radiochemotherapy, but also includes patients who previously underwent surgical treatment for tumors located from the base of the skull to the lower neck areas (including thyroid cancer).

\author{
J. A. Werner · M. Mandapathil \\ Department of Otolaryngology-Head and Neck Surgery, Philipp \\ University, Marburg, Germany \\ R. P. Takes \\ Department of Otolaryngology-Head and Neck Surgery, Radboud \\ University Medical Center, Nijmegen, The Netherlands \\ P. Strojan \\ Department of Radiation Oncology, Institute of Oncology, \\ Ljubljana, Slovenia \\ A. Rinaldo $\cdot$ A. Ferlito $(\square)$ \\ ENT Clinic, University of Udine, Piazzale S. Maria della \\ Misericordia, 33100 Udine, Italy \\ e-mail: a.ferlito@uniud.it
}


In the past, the primary treatment of head and neck cancer was radical surgical excision. But with the advent of non-surgical treatment as primary approach for organ or function preservation, surgical salvage has been considered for persistent or recurrent disease. The result is use of the term salvage surgery as a description for many different procedures [6-12]. It is clear now, that the concept of salvage surgery changes according to the initial conditions of the tumor, the initial treatment, the response to treatment, the appearance and site(s) of recurrence and their extension. Objectives and expected results will also change. Therefore, we believe that the general concept of salvage surgery should be clarified and adapted in relation to all the factors previously defined. As the term salvage surgery is used indiscriminately, attempts to summarize, compare and assess the results of different salvage surgical procedures are impeded by the heterogeneity of patients, tumors and treatments that are currently encased in the same term. Initiatives like this have also been applied in other areas as with neck dissection definitions, which have migrated from non-descriptive terms (functional, conservation neck dissection, salvage) or eponyms (Bocca, Suárez) to more descriptive terms that define, in fact, what was done and why [13].

The object of this editorial is to propose a better definition of what is and what is not salvage surgery and how different procedures can be separated and organized in a more coherent term that employs a uniform language.

The first modification is related to the term salvage. The Merrian-Webster dictionary defines salvage as "the act of saving something (such as a building, a ship, or cargo) that is in danger of being completely destroyed" or "to remove (something) from a place so that it will not be damaged, destroyed, or lost" [14]. Ideally, the application of the term to head and neck surgery should retain the same meaninga correct treatment that produced expected results initially, but for any reason has a subsequent failure, in this case a recurrence. According to this definition, the term salvage surgery should only be applied to procedures directed to patients with recurrence after successful treatment, and consequently, treatment directed to persistent tumors or lack of response to the first treatment should not be called salvage surgery. This difference is important, as in patients with persistent disease, the surgical procedures are often part of a predefined protocol to control the disease and not for "salvage", as defined above. However, it is difficult to do away with the term "salvage", in the way that it has been employed in surgical discourse for some time. Thus the term "salvage surgery" is currently used for both: a second attempt after definitive treatment, or a final attempt to cure.

Therefore, as a transition to an ideal descriptive nomenclature, currently supported by many clinicians and organizations (AAOHNS, AHNS), it may be prudent to continue to use "salvage surgery", but to qualify it by adding the reason for the operation, such as "for persistent tumor" or "for recurrent tumor" and to favor using the term "elective" and "therapeutic" neck dissection, instead of "prophylactic" or "planned" neck dissection.

As a way of understanding the differences in application of the term, first we must divide the patients according to their initial stage and draw the possible outcomes of each tumor, to define in which cases the "salvage procedure" may have a role, and offer a better terminology for it.

\section{Possible scenarios}

T1-4/N0 (local tumor with a clinically negative neck)

These tumors can be subjected to single or multimodality treatment (exclusive radiotherapy, radiochemotherapy, exclusive surgery or surgery plus radio(chemo)therapy). In any of these cases, the patient can have a complete response [or the equivalent to surgical resection with free margins (R0)] or an incomplete response (or R1-R2 resection). In case of complete response, the best scenario is cure of the disease. There is no role for further planned surgical treatment. If the patient has a recurrence, it can be an isolated local recurrence, where the role of salvage surgery will be to control the primary site ("salvage surgery" for recurrent tumor or local salvage surgery) and the surgeon may or may not choose to offer a neck dissection, which in the absence of a suspicious lymph node metastasis, should be called an elective neck dissection. If the recurrence is both local and regional (synchronous recurrence), the role of salvage surgery is to control both sites. The procedure should be named "salvage surgery" for recurrent tumor plus therapeutic (ipsilateral, contralateral or bilateral) neck dissection or locoregional salvage surgery. Finally, the recurrence can occur only in the neck (regional recurrence) where the role of salvage surgery will only be to control the neck and should be called "salvage" neck dissection for recurrent tumor or regional salvage surgery (ipsilateral, contralateral or bilateral). But the patient may also have an incomplete response [as can occur after radio(chemo)therapy] or the treatment may reveal viable tumor (as occurs with surgery with positive margins). In these cases, the surgical procedure is designed to correct the lack of response or to control the residual tumor, and it is included as a part of the treatment before starting it. Thus, it is a complement of the primary treatment. Accordingly, the procedures should be named "salvage surgery" for persistent tumor or complementary local resection, which could or could not be associated with an elective neck dissection. In this scenario, a particular characteristic of the tumor is the absence 
of nodal disease at the beginning of the treatment. It is clear that prognosis of patients with an initial N0 neck is different from those with $\mathrm{N}+$ disease. Therefore, for all salvage procedures in these patients, an $\mathrm{N}(-)$ prefix should be added.

$\mathrm{T} 1-4 / \mathrm{N}+$ (local tumor with neck lymph nodes metastasis)

These tumors can be subjected to the same variety of treatments as tumors with clinically negative necks, and may have the same variety of outcomes-with complete or incomplete responses. No further treatment is necessary in cases of complete response. Likewise, recurrences can be isolated, thus suitable for "salvage surgery" for recurrent tumor or local salvage surgery and the surgeon may or may not choose to offer a neck dissection, which in the absence of a suspicious lymph nodes metastasis, should be called an elective neck dissection. However, in the case of $\mathrm{N}+$ disease the recurrence can be local and regional (synchronous recurrence) and the role of salvage surgery is to control both sites. Subsequent surgical treatment should be termed "salvage surgery" for recurrent tumor plus therapeutic neck dissection or locoregional salvage surgery. Finally, the recurrence may occur only in the neck (regional recurrence) where the role of salvage surgery will only be to control the neck and should be called "salvage" neck dissection for recurrent tumor or regional salvage surgery. The patient can also have an incomplete response (as can occur after radiochemotherapy) or the treatment may identify viable tumor (surgery with positive margins). In these cases, the neck can achieve a complete response but tumor may persist at the primary site, therefore requiring "salvage surgery" for persistent tumor or complementary local resection, associated or not with an elective neck dissection. These cases correspond to the previously termed "planned neck dissection" when the patient presented pretreatment neck metastasis which achieved a complete response, but the surgeon performed the neck dissection based on the pretreatment tumor stage. In other cases the primary tumor may have a complete response but the neck does not. In this situation, the surgical procedure is focused on controlling neck tumor, and this corresponds to a "salvage" neck dissection for persistent tumor or complementary therapeutic neck dissection. Finally, the patient can have persistence of the tumor at both the primary site and regional lymph nodes, and in this case the surgery corresponds to a "salvage surgery" for persistent tumor or complementary local resection associated with a therapeutic neck dissection. In this category for all salvage procedures an $\mathrm{N}(+)$ prefix should be added. According to what was discussed earlier, the term "surgery" or "local" should be changed for the specific name of the surgical procedure (e.g. salvage laryngectomy for persistent tumor or complementary laryngectomy).

Finally, a selected group of patients with isolated distant metastasis to the lungs have been considered candidates for pulmonary resection. The criteria and indications are single or a few resectable metastases in patients without any evidence of locoregional disease or other foci of distant metastases. This group can be called "salvage surgery" for isolated metastasis. Secondary tumors following primary radio(chemo)therapy, which may arise in a secondary "field cancerization" or as a radiation-induced malignancy or as a de novo process often may be mistaken for local recurrence when arising in close proximity to the primary tumor, and should therefore not be considered as salvage surgery. However, as already mentioned, a distinction between local recurrence and secondary tumor may be difficult.

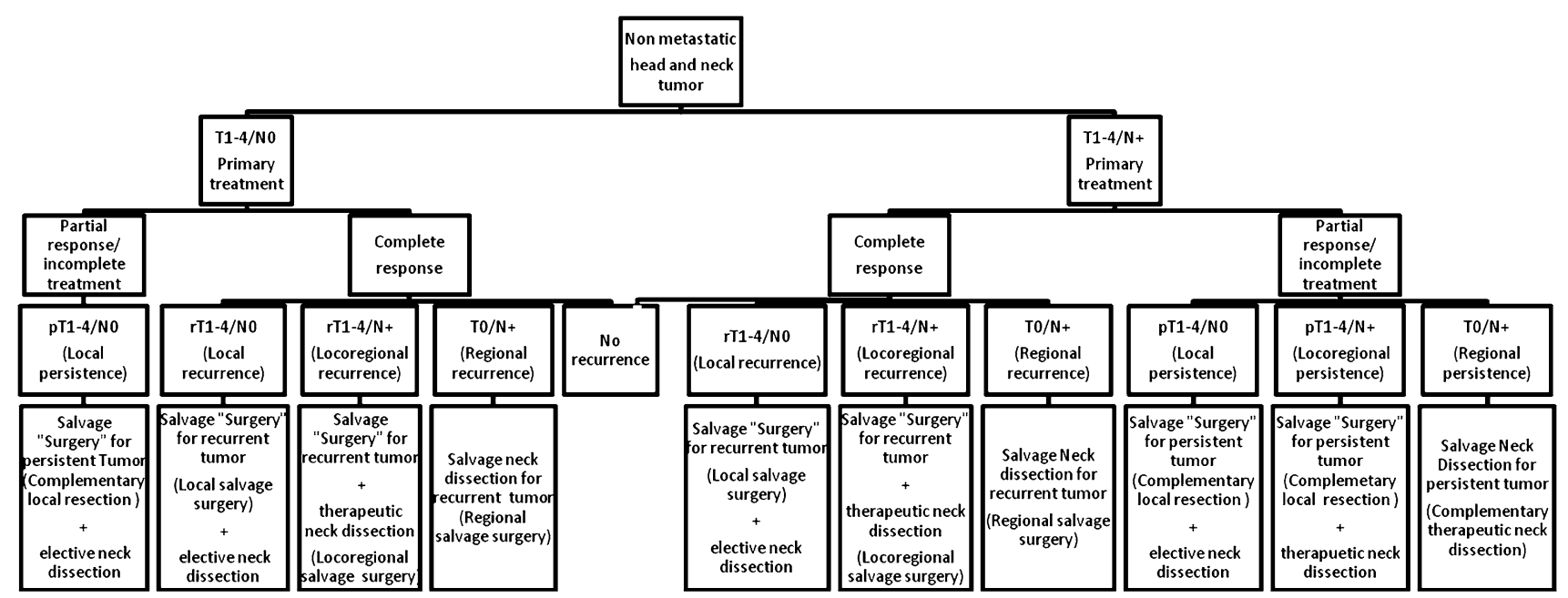

Fig. 1 Fate of head and neck cancer patients and definitions for "salvage" approach. Asterisk persistence: tumor detected within 3 months of completion of treatment; recurrence: tumor detected after 3 months of completion of treatment 


\section{Cancer of unknown primary (CUP)-syndrome}

Early stages of these tumors can be treated with surgery alone or radiotherapy alone resulting in a complete or partial response. N2a-N3 CUP syndromes are treated with primary surgery and postoperative radio(chemo)therapy or definite radiochemotherapy. In case of recurrence, the surgical procedure corresponds to a therapeutic salvage neck dissection for recurrent disease. In case of tumor persistence in partial response, the procedure should be called therapeutic salvage neck dissection for persisting disease.

When the surgeon chooses to offer surgery following radio(chemo)therapy routinely (in the absence of evident disease), the procedure should be referred to as elective salvage neck dissection. If following neck surgery and/or radio(chemo)therapy a tumor develops at a primary local site (other than the neck), therapeutic salvage surgery is applied with or without an elective neck dissection in negative necks, or including a therapeutics salvage neck dissection in case of concomitant regional recurrence. However, a differentiation between persistence and recurrence is often not appraisable in this special situation.

This revised terminology helps to distinguish between different procedures with different objectives and different prognoses, which often are combined and analyzed together (Fig. 1). This distinction will help to understand the effects of each of these surgical procedures and to offer a better datum to analyze their advantages and weaknesses.

\section{References}

1. Tupper C (1965) Radical Wertheim as a salvage procedure. Patients with recurrence following initial definitive radiotherapy. Am J Obstet Gynecol 91:364-368

2. Poncet $P$ (1971) Effects of tumor localization in the prognosis of salvage laryngectomies. Ann Otolaryngol Chir Cervicofac 88:355-356 (article in French)

3. Poncet P (1975) Total laryngectomy for salvage in cancers of the glottic region. Laryngoscope 85:1430-1434
4. Kowalski LP, Rinaldo A, Robbins KT, Pellitteri PK, Shaha AR, Weber RS, Ferlito A (2003) Stomal recurrence: pathophysiology, treatment and prevention. Acta Otolaryngol 123:421-432

5. Younes RN, Gross JL, Silva JF, Fernandez JA, Kowalski LP (1997) Surgical treatment of lung metastases of head and neck tumors. Am J Surg 174:499-502

6. Arce K, Bell RB, Potter JK, Buehler MJ, Potter BE, Dierks EJ (2012) Vascularized free tissue transfer for reconstruction of ablative defects in oral and oropharyngeal cancer patients undergoing salvage surgery following concomitant chemoradiation. Int J Oral Maxillofac Surg 41:733-738

7. Dequanter D, Lothaire P (2008) The role of salvage surgery in organ preservation strategies in advanced head and neck cancer. B-ENT 4:77-80

8. Hasney CP, Swanton RG, Friedlander PL (2010) CyberKnife stereotactic radiosurgery for recurrent squamous cell carcinoma of the head and neck following salvage surgery with close or positive margins. Laryngoscope 120(Suppl 4):S152

9. Lee DJ, Kwon KH, Chung EJ, Park IS, Kim JH, Rho YS (2013) The role of elective neck dissection during salvage surgery in head and neck squamous cell carcinoma. Acta Otolaryngol 133:886-892

10. McLaughlin MP, Parsons JT, Fein DA, Stringer SP, Cassisi NJ, Mendenhall WM, Million RR (1996) Salvage surgery after radiotherapy failure in T1-T2 squamous cell carcinoma of the glottic larynx. Head Neck 18:229-235

11. Spriano G, Pellini R, Romano G, Muscatello L, Roselli R (2002) Supracricoid partial laryngectomy as salvage surgery after radiation failure. Head Neck 24:759-765

12. White H, Ford S, Bush B, Holsinger FC, Moore E, Ghanem T, Carroll W, Rosenthal E, Magnuson JS (2013) Salvage surgery for recurrent cancers of the oropharynx: comparing TORS with standard open surgical approaches. JAMA Otolaryngol Head Neck Surg 139:773-778

13. Ferlito A, Robbins KT, Shah JP, Medina JE, Silver CE, AlTamimi S, Fagan JJ, Paleri V, Takes RP, Bradford CR, Devaney KO, Stoeckli SJ, Weber RS, Bradley PJ, Suárez C, Leemans CR, Coskun HH, Pitman KT, Shaha AR, de Bree R, Hartl DM, Haigentz M Jr, Rodrigo JP, Hamoir M, Khafif A, Langendijk JA, Owen RP, Sanabria A, Strojan P, Vander Poorten V, Werner JA, Bień S, Woolgar JA, Zbären P, Betka J, Folz BJ, Genden EM, Talmi YP, Strome M, González Botas JH, Olofsson J, Kowalski LP, Holmes JD, Hisa Y, Rinaldo A (2011) Proposal for a rational classification of neck dissections. Head Neck 33:445-450

14. Encyclopedia Britannica (2014) Merriam-Webster. http://www. merriam-webster.com/dictionary/salvage. Accessed 20 Jan 2014 